Supplement of Biogeosciences, 13, 6487-6505, 2016

http://www.biogeosciences.net/13/6487/2016/

doi:10.5194/bg-13-6487-2016-supplement

(C) Author(s) 2016. CC Attribution 3.0 License.

(c) (i)

Supplement of

\title{
Carbonate "clumped" isotope signatures in aragonitic scleractinian and calcitic gorgonian deep-sea corals
}

Justine Kimball et al.

Correspondence to: Robert Eagle (robeagle@g.ucla.edu)

The copyright of individual parts of the supplement might differ from the CC-BY 3.0 licence. 
Table S1: Raw data for equilibrated gases, standards, and samples.

Figure S1: Equilibrated gas lines for February 2013.

Figure S2: Equilibrated gas lines for May-July 2013.

All data collected at UCLA 


\begin{tabular}{|c|c|c|c|c|c|c|c|c|c|c|c|c|c|c|c|}
\hline Counter & Date & $\begin{array}{l}\text { Sample } \\
\text { ID }\end{array}$ & spec \#'s & $\begin{array}{l}\mathrm{d} 13 \mathrm{C} \\
\text { (PDB) }\end{array}$ & $\begin{array}{l}\text { d13C } \\
\text { stdev }\end{array}$ & $\begin{array}{l}\text { d180 gas } \\
\text { (SMOW) }\end{array}$ & $\begin{array}{l}\text { d180 } \\
\text { stdev }\end{array}$ & $\begin{array}{c}\mathrm{d} 47 \\
(\mathrm{v} . \mathrm{Oz})\end{array}$ & $\begin{array}{c}\text { d47 } \\
\text { stdev }\end{array}$ & $\begin{array}{c}\text { D47 } \\
\text { (v. Oz) }\end{array}$ & $\begin{array}{l}\text { D47 } \\
\text { stdev }\end{array}$ & $\begin{array}{l}\text { D47 } \\
\text { sterror }\end{array}$ & $\begin{array}{c}d 48 \\
\text { (v. Oz) }\end{array}$ & $\begin{array}{c}\text { D48 } \\
\text { (v. Oz) }\end{array}$ & $\begin{array}{l}\text { D48 } \\
\text { stdev }\end{array}$ \\
\hline \multirow{10}{*}{ high voltage bellows overcomp? } & $02 / 07 / 12$ & TV01 & 3160 & 2.271 & 0.004 & 29.996 & 0.014 & 10.527 & 0.016 & -0.149 & 0.011 & 0.004 & 10.687 & 0.930 & 0.132 \\
\hline & $2 / 1 / 13$ & 86 Breakseal heated $\mathrm{CO} 2$ tank & 2919 & -37.281 & 0.003 & 3.260 & 0.027 & -54.485 & 0.042 & -1.076 & 0.031 & 0.011 & -44.669 & -2.673 & 0.122 \\
\hline & $02 / 02 / 13$ & 102-GC-A201 & 2935 & 0.335 & 0.004 & 23.967 & 0.009 & 2.561 & 0.030 & -0.221 & 0.024 & 0.009 & -2.029 & 0.005 & 0.092 \\
\hline & $2 / 2 / 13$ & 69 Heated reg DI+tank CO2 & 2964 & -28.438 & 0.006 & 24.745 & 0.020 & -25.170 & 0.022 & -0.942 & 0.022 & 0.008 & -0.682 & -0.036 & 0.096 \\
\hline & $2 / 2 / 13$ & 66 Unheated Oztech & 2973 & -3.863 & 0.018 & 23.614 & 0.024 & -1.561 & 0.050 & 0.062 & 0.019 & 0.007 & -2.547 & 0.192 & 0.099 \\
\hline & $2 / 2 / 13$ & 64 heated Oztech & 2981 & -3.671 & 0.002 & 22.220 & 0.008 & -3.631 & 0.044 & -0.809 & 0.041 & 0.015 & -5.862 & -0.413 & 0.146 \\
\hline & $02 / 03 / 13$ & Carmel Chalk & 3005 & -2.371 & 0.003 & 34.599 & 0.010 & 10.567 & 0.019 & -0.182 & 0.014 & 0.005 & 20.348 & 1.557 & 0.112 \\
\hline & $2 / 3 / 13$ & Breakseal 92 heated tank & 3022 & -28.066 & 0.002 & 23.965 & 0.009 & -25.550 & 0.036 & -0.925 & 0.038 & 0.013 & -2.099 & 0.064 & 0.094 \\
\hline & $2 / 3 / 13$ & Breakseal 67 Oztech $25 \mathrm{C}$ & 3030 & -3.263 & 0.003 & 26.693 & 0.008 & 2.055 & 0.026 & 0.037 & 0.019 & 0.007 & 3.605 & 0.338 & 0.112 \\
\hline & $2 / 3 / 13$ & 78 reg DI +tank $\mathrm{CO} 2$ heated & 3039 & -28.013 & 0.004 & 26.854 & 0.009 & -22.704 & 0.030 & -0.933 & 0.030 & 0.011 & 3.667 & 0.197 & 0.082 \\
\hline \multirow[t]{22}{*}{ D47 and D48 are high } & $2 / 6 / 13$ & 79 heated tank $\mathrm{CO} 2+$ reg $\mathrm{DI}$ & 3126 & -28.061 & 0.003 & 27.808 & 0.018 & -21.742 & 0.054 & -0.848 & 0.043 & 0.015 & 6.496 & 1.158 & 0.327 \\
\hline & $2 / 6 / 13$ & 75 heated tank $\mathrm{CO} 2+$ reg $\mathrm{DI}$ & 3140 & -28.460 & 0.004 & 24.944 & 0.018 & -25.020 & 0.039 & -0.965 & 0.025 & 0.009 & -0.326 & -0.068 & 0.167 \\
\hline & $02 / 08 / 13$ & 102-GC-A201 & 3205 & 0.247 & 0.003 & 23.849 & 0.017 & 2.382 & 0.018 & -0.196 & 0.023 & 0.008 & -1.997 & 0.268 & 0.186 \\
\hline & $2 / 8 / 13$ & 98 Seawater+Oztech & 3240 & -3.951 & 0.002 & 34.956 & 0.013 & 9.633 & 0.019 & 0.068 & 0.024 & 0.008 & 21.082 & 1.593 & 0.130 \\
\hline & $02 / 08 / 13$ & Fast HAGA & 3254 & 3.044 & 0.002 & 32.861 & 0.008 & 14.117 & 0.035 & -0.176 & 0.032 & 0.011 & 16.607 & 1.217 & 0.084 \\
\hline & $02 / 09 / 13$ & Carmel Chalk & 3262 & -2.290 & 0.003 & 34.502 & 0.010 & 10.529 & 0.035 & -0.202 & 0.029 & 0.010 & 20.129 & 1.529 & 0.157 \\
\hline & $02 / 13 / 13$ & 102-GC-AZ01 & 3477 & 0.278 & 0.005 & 23.894 & 0.018 & 2.532 & 0.064 & -0.121 & 0.048 & 0.017 & -1.649 & 0.529 & 0.190 \\
\hline & $2 / 13 / 13$ & $509-\mathrm{CO} 2-5.99 \mathrm{mB}-\mathrm{H}$ & 3497 & -37.384 & 0.004 & 4.568 & 0.009 & -53.438 & 0.018 & -1.194 & 0.017 & 0.006 & -42.476 & -2.983 & 0.149 \\
\hline & $2 / 14 / 13$ & 100 Bone dry CO2 $5.86 \mathrm{UH}$ & 3542 & -37.868 & 0.005 & 2.671 & 0.014 & -54.834 & 0.028 & -0.259 & 0.026 & 0.009 & -45.774 & -2.656 & 0.155 \\
\hline & $02 / 14 / 13$ & Carmel Chalk & 3555 & -2.244 & 0.008 & 34.670 & 0.013 & 10.758 & 0.020 & -0.186 & 0.014 & 0.005 & 20.499 & 1.567 & 0.153 \\
\hline & $02 / 15 / 13$ & Carrera Marble & 3590 & 2.285 & 0.003 & 37.307 & 0.017 & 17.608 & 0.035 & -0.388 & 0.030 & 0.011 & 25.971 & 1.808 & 0.271 \\
\hline & $02 / 15 / 13$ & 101 bone dry tank CO2 - heated & 3611 & -37.456 & 0.001 & 4.424 & 0.005 & -53.630 & 0.016 & -1.179 & 0.018 & 0.006 & -42.784 & -3.018 & 0.072 \\
\hline & $02 / 16 / 13$ & $146-6$ & 3636 & -2.832 & 0.004 & 40.636 & 0.013 & 16.252 & 0.043 & -0.057 & 0.035 & 0.012 & 33.021 & 2.279 & 0.158 \\
\hline & $2 / 16 / 13$ & bone dry tank $\mathrm{CO} 2$ - heated & 3648 & -37.478 & 0.003 & 3.589 & 0.019 & -54.450 & 0.029 & -1.176 & 0.034 & 0.012 & -44.443 & -3.091 & 0.186 \\
\hline & $02 / 16 / 13$ & 513 bone dry tank $\mathrm{CO} 2$ - heated & 3648 & -37.478 & 0.003 & 3.590 & 0.018 & -54.443 & 0.026 & -1.171 & 0.035 & 0.012 & -44.436 & -3.086 & 0.193 \\
\hline & $2 / 16 / 13$ & 501 Evap DI + Oz H & 3656 & -3.949 & 0.007 & 40.853 & 0.013 & 14.740 & 0.024 & -0.679 & 0.029 & 0.010 & 33.330 & 2.166 & 0.146 \\
\hline & $2 / 16 / 13$ & 102 Bonedry CO2 heated & 3664 & -38.482 & 0.006 & 2.197 & 0.022 & -56.741 & 0.035 & -1.184 & 0.024 & 0.009 & -47.100 & -3.098 & 0.181 \\
\hline & $2 / 16 / 13$ & TV-01 & 3676 & 2.294 & 0.004 & 30.154 & 0.011 & 10.703 & 0.030 & -0.154 & 0.024 & 0.008 & 11.044 & 0.976 & 0.144 \\
\hline & $2 / 17 / 13$ & 103 Bonedry heated & 3697 & -37.467 & 0.007 & 3.826 & 0.024 & -54.212 & 0.040 & -1.175 & 0.030 & 0.011 & -43.748 & -2.836 & 0.152 \\
\hline & $2 / 18 / 13$ & 514 Bonedry $\mathrm{CO} 26 \mathrm{mB}-\mathrm{UH}$ & 3762 & -37.547 & 0.002 & 3.019 & 0.006 & -54.195 & 0.026 & -0.259 & 0.025 & 0.009 & -45.090 & -2.634 & 0.044 \\
\hline & $2 / 18 / 13$ & 106 Bonedry $\mathrm{CO} 25.55 \mathrm{mB} \mathrm{H}$ & 3770 & -37.311 & 0.007 & 3.378 & 0.009 & -54.462 & 0.030 & -1.141 & 0.021 & 0.007 & -44.639 & -2.877 & 0.252 \\
\hline & $2 / 18 / 13$ & $694-3$ & 3778 & -1.129 & 0.002 & 38.977 & 0.009 & 16.186 & 0.036 & -0.139 & 0.029 & 0.010 & 29.507 & 2.052 & 0.132 \\
\hline GC column changed & $2 / 19 / 13$ & Antartic Mollusc & 3786 & 1.737 & 0.003 & 43.283 & 0.007 & 23.422 & 0.025 & -0.017 & 0.024 & 0.008 & 38.687 & 2.655 & 0.186 \\
\hline \multirow[t]{4}{*}{ GC column changed } & $2 / 19 / 13$ & Antartic Mollusc & 3786 & 1.737 & 0.003 & 43.283 & 0.007 & 23.422 & 0.025 & -0.017 & 0.024 & 0.008 & 38.687 & 2.655 & 0.186 \\
\hline & $02 / 19 / 13$ & 108 Bonedry CO2 $5.5 \mathrm{mB}$ UH & 3797 & -37.060 & 0.002 & 3.117 & 0.014 & -53.599 & 0.030 & -0.215 & 0.027 & 0.010 & -44.684 & -2.407 & 0.206 \\
\hline & $2 / 20 / 13$ & Spel-2-8E & 3805 & -9.571 & 0.003 & 32.409 & 0.005 & 1.300 & 0.034 & -0.260 & 0.032 & 0.011 & 15.935 & 1.486 & 0.076 \\
\hline & $02 / 20 / 13$ & 102-GC-AZ01 & 3813 & 0.443 & 0.002 & 23.759 & 0.008 & 2.490 & 0.025 & -0.187 & 0.025 & 0.009 & -2.315 & 0.123 & 0.149 \\
\hline
\end{tabular}




\begin{tabular}{|c|c|c|c|c|c|c|c|c|c|c|c|c|c|c|}
\hline $02 / 20 / 13$ & $90 \mathrm{CO} 2 \operatorname{tank} 6.11 \mathrm{mB} \mathrm{H}$ & 3821 & -28.194 & 0.003 & 24.274 & 0.006 & -25.437 & 0.026 & -0.989 & 0.020 & 0.007 & -1.723 & -0.160 & 124 \\
\hline 2/20/13 & $3808-4$ & 3831 & -2.907 & 0.003 & 40.032 & 0.004 & 15.499 & 0.032 & -0.133 & 0.028 & 0.010 & 31.761 & 2.219 & 0.086 \\
\hline 2/20/13 & Antarctic Mollusc & 3839 & 1.774 & 0.003 & 43.241 & 0.012 & 23.421 & 0.046 & -0.014 & 0.040 & 0.014 & 38.774 & 2.818 & 0.096 \\
\hline 2/20/13 & Antarctic Mollusc & 3839 & 1.774 & 0.003 & 43.241 & 0.012 & 23.421 & 0.046 & -0.014 & 0.040 & 0.014 & 38.774 & 2.818 & 0.096 \\
\hline $2 / 21 / 13$ & 516 Oz+Evap DI H & 3847 & -3.683 & 0.002 & 42.620 & 0.008 & 16.722 & 0.032 & -0.713 & 0.025 & 0.009 & 36.839 & 2.166 & 0.182 \\
\hline $2 / 21 / 13$ & $146-6$ & 3855 & -2.663 & 0.004 & 40.600 & 0.010 & 16.367 & 0.032 & -0.072 & 0.027 & 0.010 & 32.925 & 2.254 & 0.200 \\
\hline $2 / 21 / 13$ & $148-2$ & 3863 & -2.409 & 0.004 & 40.257 & 0.009 & 16.307 & 0.024 & -0.040 & 0.022 & 0.008 & 32.179 & 2.190 & 0.098 \\
\hline $2 / 21 / 13$ & 110 Evap DI+oz UH & 3872 & -3.600 & 0.005 & 44.962 & 0.011 & 19.955 & 0.032 & 0.095 & 0.024 & 0.008 & 42.209 & 2.851 & 0.135 \\
\hline $2 / 21 / 13$ & 518 Bonedry UH & 3882 & -37.295 & 0.003 & 3.233 & 0.012 & -53.723 & 0.017 & -0.229 & 0.017 & 0.006 & -44.582 & -2.529 & 0.126 \\
\hline $2 / 21 / 13$ & 519 Bonedry UH & 3891 & -37.340 & 0.003 & 3.403 & 0.007 & -53.645 & 0.022 & -0.276 & 0.030 & 0.011 & -44.352 & -2.627 & 0.124 \\
\hline 2/22/13 & $592-1$ & 3916 & -2.390 & 0.003 & 39.210 & 0.006 & 15.203 & 0.028 & -0.119 & 0.025 & 0.009 & 30.210 & 2.292 & 0.158 \\
\hline $2 / 22 / 13$ & $3808-4$ & 3924 & -2.914 & 0.004 & 40.120 & 0.022 & 15.622 & 0.027 & -0.092 & 0.024 & 0.008 & 32.191 & 2.467 & 0.166 \\
\hline $2 / 22 / 13$ & $520 \mathrm{CO} 2$ bonedry UH & 3933 & -37.709 & 0.003 & 3.227 & 0.018 & -47.380 & 0.037 & -0.227 & 0.095 & 0.034 & -44.620 & -2.236 & 0.911 \\
\hline $2 / 22 / 13$ & 109 Oz+Evap DI H & 3941 & -3.834 & 0.002 & 42.474 & 0.007 & 16.419 & 0.016 & -0.723 & 0.016 & 0.006 & 36.519 & 2.137 & 0.094 \\
\hline $2 / 22 / 13$ & $522 \mathrm{CO} 2$ Heated & 3952 & -37.790 & 0.005 & 4.074 & 0.009 & -54.207 & 0.030 & -1.100 & 0.034 & 0.012 & -42.845 & -2.386 & 0.158 \\
\hline $2 / 22 / 13$ & Carrera Marble & 3961 & 2.193 & 0.002 & 36.988 & 0.006 & 17.196 & 0.019 & -0.390 & 0.018 & 0.006 & 25.395 & 1.863 & 0.105 \\
\hline 2/23/13 & $703-2$ & 3969 & -1.873 & 0.009 & 41.513 & 0.015 & 18.100 & 0.039 & -0.024 & 0.041 & 0.014 & 35.081 & 2.585 & 0.153 \\
\hline $2 / 23 / 13$ & $502 \mathrm{CO} 2$ tank Heated & 3988 & -37.459 & 0.002 & 4.289 & 0.011 & -53.765 & 0.028 & -1.182 & 0.032 & 0.011 & -42.898 & -2.869 & 0.088 \\
\hline 2/23/13 & 115 Oz+Evap DI UH & 3999 & -3.913 & 0.002 & 47.415 & 0.004 & 22.174 & 0.040 & 0.181 & 0.039 & 0.014 & 47.467 & 3.201 & 0.111 \\
\hline $2 / 23 / 13$ & TV01 & 4007 & 2.464 & 0.004 & 30.063 & 0.006 & 10.774 & 0.023 & -0.157 & 0.019 & 0.007 & 10.794 & 0.905 & 0.087 \\
\hline $02 / 23 / 13$ & Spel 2-8-E & 4016 & -9.518 & 0.002 & 32.299 & 0.006 & 1.263 & 0.027 & -0.240 & 0.024 & 0.008 & 15.491 & 1.261 & 0.117 \\
\hline $2 / 24 / 13$ & 703-5 outer & 4032 & -4.759 & 0.004 & 38.879 & 0.009 & 12.528 & 0.039 & -0.143 & 0.031 & 0.011 & 29.152 & 1.910 & 0.126 \\
\hline $2 / 24 / 13$ & $3808-5$ & 4040 & -5.606 & 0.003 & 39.128 & 0.008 & 11.957 & 0.022 & -0.132 & 0.023 & 0.008 & 29.765 & 2.030 & 0.125 \\
\hline $2 / 24 / 13$ & $3806-1$ & 4048 & -4.139 & 0.002 & 39.669 & 0.007 & 13.932 & 0.033 & -0.132 & 0.034 & 0.012 & 30.987 & 2.170 & 0.117 \\
\hline $2 / 24 / 13$ & $14 \mathrm{Oz}+$ Evap heated & 4057 & -3.748 & 0.003 & 48.058 & 0.008 & 23.002 & 0.032 & 0.206 & 0.031 & 0.011 & 49.165 & 3.596 & 0.175 \\
\hline $02 / 24 / 13$ & Carrera Marble & 4065 & 2.239 & 0.003 & 36.980 & 0.008 & 17.192 & 0.026 & -0.431 & 0.022 & 0.008 & 25.234 & 1.720 & 0.129 \\
\hline $2 / 24 / 13$ & $3803-3$ & 4074 & -4.619 & 0.004 & 39.383 & 0.006 & 13.169 & 0.026 & -0.140 & 0.026 & 0.009 & 30.395 & 2.148 & 0.114 \\
\hline $2 / 25 / 13$ & Carrera Marble & 4110 & 2.282 & 0.002 & 36.930 & 0.010 & 17.179 & 0.023 & -0.436 & 0.023 & 0.008 & 24.937 & 1.527 & 0.144 \\
\hline $2 / 25 / 13$ & 523 & 4127 & -37.387 & 0.004 & 4.131 & 0.015 & -53.818 & 0.010 & -1.150 & 0.023 & 0.008 & -43.073 & -2.738 & 0.151 \\
\hline $02 / 26 / 13$ & 102-GC-AZ01 & 4143 & 0.436 & 0.004 & 23.829 & 0.018 & 2.543 & 0.034 & -0.198 & 0.037 & 0.013 & -1.750 & 0.553 & 0.101 \\
\hline $2 / 26 / 13$ & 524-tank-heated & 4159 & -37.277 & 0.002 & 4.588 & 0.003 & -53.312 & 0.027 & -1.189 & 0.029 & 0.010 & -42.233 & -2.771 & 0.196 \\
\hline $2 / 26 / 13$ & Fast HAGA & 4168 & 3.068 & 0.002 & 32.765 & 0.004 & 14.047 & 0.020 & -0.174 & 0.022 & 0.008 & 16.384 & 1.184 & 0.118 \\
\hline $2 / 26 / 13$ & Carmel Chalk & 4176 & -2.155 & 0.002 & 34.640 & 0.008 & 10.810 & 0.022 & -0.191 & 0.024 & 0.009 & 20.319 & 1.448 & 0.181 \\
\hline $2 / 26 / 13$ & 703-5 outer & 4184 & -4.923 & 0.003 & 38.834 & 0.006 & 12.339 & 0.044 & -0.128 & 0.046 & 0.016 & 29.173 & 2.017 & 0.075 \\
\hline $2 / 27 / 13$ & 116-Oz+Evap-5.7mB-U & 4221 & -3.616 & 0.004 & 48.473 & 0.005 & 23.512 & 0.019 & 0.172 & 0.022 & 0.008 & 49.576 & 3.194 & 0.068 \\
\hline $2 / 27 / 13$ & $526 \mathrm{CO} 25.54 \mathrm{mB} \cup \mathrm{H}$ & 4231 & -32.547 & 13.356 & 7.392 & 11.976 & -39.468 & 24.558 & -0.222 & 0.093 & 0.033 & -35.971 & -1.752 & 1.730 \\
\hline $02 / 28 / 13$ & TV01 & 4241 & 2.468 & 0.002 & 30.095 & 0.004 & 10.804 & 0.022 & -0.164 & 0.020 & 0.007 & 10.863 & 0.911 & 0.137 \\
\hline $2 / 28 / 13$ & 112 Evap DI + Oz 5.91 mB UH & 4266 & -3.648 & 0.003 & 48.587 & 0.006 & 23.598 & 0.024 & 0.178 & 0.022 & 0.008 & 49.813 & 3.204 & 0.089 \\
\hline 2/28/13 & 118 Bonedry CO2 $5.97 \mathrm{UH}$ & 4275 & -37.415 & 0.006 & 3.076 & 0.015 & -53.939 & 0.022 & -0.179 & 0.024 & 0.008 & -44.623 & -2.261 & 0.437 \\
\hline
\end{tabular}




\begin{tabular}{|c|c|c|c|c|c|c|c|c|c|c|c|c|c|c|c|}
\hline & $2 / 28 / 13$ & Antarctic mollusc & 4285 & 1.750 & 0.003 & 43.257 & 0.003 & 23.385 & 0.030 & -0.040 & 0.030 & 0.011 & 38.497 & 2.521 & 0.138 \\
\hline & $03 / 01 / 13$ & 47407 coral & 4293 & -1.976 & 0.003 & 41.513 & 0.010 & 17.978 & 0.019 & -0.044 & 0.018 & 0.006 & 34.749 & 2.264 & 0.098 \\
\hline & $3 / 1 / 13$ & 703-2 & 4301 & -2.056 & 0.002 & 41.435 & 0.014 & 17.838 & 0.029 & -0.029 & 0.032 & 0.011 & 34.712 & 2.377 & 0.091 \\
\hline \multirow[t]{4}{*}{ *only 7 acquisitions! } & $3 / 1 / 13$ & 705-3 inner & 4309 & -7.683 & 0.002 & 37.877 & 0.014 & 7.586 & 0.021 & -0.126 & 0.053 & 0.019 & 27.106 & 1.629 & 0.669 \\
\hline & $3 / 1 / 13$ & 533 Evap DI + Oz UH 6.02 mB & 4317 & -4.397 & 0.003 & 44.413 & 0.004 & 18.692 & 0.026 & 0.161 & 0.026 & 0.009 & 40.912 & 2.658 & 0.084 \\
\hline & $3 / 1 / 13$ & $\mathrm{CM}$ & 4325 & 2.247 & 0.002 & 36.967 & 0.009 & 17.157 & 0.028 & -0.460 & 0.027 & 0.010 & 25.083 & 1.597 & 0.112 \\
\hline & $3 / 1 / 13$ & 534 Evap DI+Oz UH $5.86 \mathrm{mB}$ & 4334 & -3.756 & 0.003 & 44.458 & 0.006 & 19.384 & 0.021 & 0.177 & 0.021 & 0.007 & 41.201 & 2.847 & 0.045 \\
\hline \multirow[t]{3}{*}{ changed acid } & $3 / 1 / 13$ & 102-GC-AZOL & 4343 & 0.550 & 0.003 & 23.955 & 0.009 & 2.787 & 0.034 & -0.189 & 0.032 & 0.011 & -1.816 & 0.242 & 0.236 \\
\hline & $3 / 2 / 13$ & spel-2-8-E & 4351 & -9.775 & 0.003 & 32.372 & 0.010 & 1.081 & 0.009 & -0.244 & 0.011 & 0.004 & 15.704 & 1.331 & 0.108 \\
\hline & $3 / 2 / 13$ & $592-1$ & 4367 & -2.448 & 0.006 & 39.069 & 0.009 & 14.997 & 0.026 & -0.128 & 0.023 & 0.008 & 29.884 & 2.246 & 0.680 \\
\hline \multirow[t]{17}{*}{$\mathrm{V}$ too low??/7 acquisitions } & $3 / 2 / 13$ & 535 Evap DI+Oz UH 5.51 mB & 4375 & -3.444 & 0.002 & 43.420 & 0.010 & 16.340 & 0.042 & 0.168 & 0.077 & 0.027 & 39.225 & 2.567 & 1.045 \\
\hline & $3 / 2 / 13$ & 123 Bonedry UH $6.21 \mathrm{mB}$ & 4383 & -37.533 & 0.003 & 3.576 & 0.010 & -53.721 & 0.021 & -0.338 & 0.024 & 0.008 & -44.141 & -2.750 & 0.055 \\
\hline & $3 / 2 / 13$ & $148-2$ & 4392 & -2.724 & 0.002 & 40.131 & 0.003 & 15.842 & 0.032 & -0.070 & 0.029 & 0.010 & 31.741 & 2.008 & 0.094 \\
\hline & $3 / 2 / 13$ & $146-6$ & 4400 & -2.511 & 0.004 & 40.606 & 0.009 & 16.555 & 0.016 & -0.039 & 0.016 & 0.006 & 32.968 & 2.283 & 0.116 \\
\hline & $3 / 3 / 13$ & Carmel Chalk & 4408 & -2.157 & 0.003 & 34.656 & 0.005 & 10.818 & 0.027 & -0.197 & 0.027 & 0.009 & 20.371 & 1.466 & 0.081 \\
\hline & $3 / 3 / 13$ & 703-5 outer & 4426 & -5.160 & 0.003 & 38.788 & 0.008 & 12.057 & 0.032 & -0.131 & 0.029 & 0.010 & 28.965 & 1.905 & 0.109 \\
\hline & $3 / 3 / 13$ & $3804-4$ & 4444 & -2.982 & 0.002 & 40.003 & 0.010 & 15.419 & 0.026 & -0.112 & 0.028 & 0.010 & 31.658 & 2.174 & 0.160 \\
\hline & $3 / 3 / 13$ & $\mathrm{CM}$ & 4453 & 2.206 & 0.004 & 37.046 & 0.012 & 17.222 & 0.030 & -0.434 & 0.027 & 0.010 & 25.365 & 1.720 & 0.149 \\
\hline & $3 / 4 / 13$ & $3808-1$ & 4462 & -3.198 & 0.003 & 39.745 & 0.012 & 14.935 & 0.029 & -0.127 & 0.032 & 0.011 & 31.221 & 2.249 & 0.164 \\
\hline & $3 / 4 / 13$ & $3808-5$ & 4471 & -6.013 & 0.003 & 39.026 & 0.012 & 11.461 & 0.029 & -0.128 & 0.023 & 0.008 & 29.578 & 2.048 & 0.135 \\
\hline & $3 / 4 / 13$ & Carrer Marble & 4480 & 2.163 & 0.003 & 36.911 & 0.015 & 17.055 & 0.024 & -0.425 & 0.025 & 0.009 & 25.205 & 1.827 & 0.172 \\
\hline & $3 / 4 / 13$ & 119 Bonedry UH & 4489 & -37.327 & 0.003 & 3.672 & 0.010 & -53.338 & 0.036 & -0.237 & 0.034 & 0.012 & -44.017 & -2.812 & 0.095 \\
\hline & $3 / 4 / 13$ & 120 Bonedry H & 4498 & -37.738 & 0.002 & 3.811 & 0.007 & -54.368 & 0.025 & -1.055 & 0.025 & 0.009 & -43.308 & -2.347 & 0.122 \\
\hline & $03 / 04 / 13$ & 125 Evap DI Oztech UH & 4510 & -13.785 & 0.003 & 32.252 & 0.007 & -2.596 & 0.029 & 0.101 & 0.035 & 0.012 & 15.529 & 1.410 & 0.136 \\
\hline & $3 / 5 / 13$ & 527 tank CO2 - Unheated & 4526 & -37.519 & 0.003 & 2.456 & 0.008 & -54.713 & 0.022 & -0.262 & 0.021 & 0.007 & -46.330 & -2.811 & 0.225 \\
\hline & $3 / 6 / 13$ & SPEL-28-E & 4545 & -9.068 & 0.003 & 32.378 & 0.009 & 1.793 & 0.023 & -0.226 & 0.024 & 0.008 & 15.557 & 1.171 & 0.196 \\
\hline & $3 / 6 / 13$ & $3808-41$ & 4553 & -2.955 & 0.005 & 40.296 & 0.018 & 15.767 & 0.033 & -0.083 & 0.029 & 0.010 & 32.307 & 2.241 & 0.187 \\
\hline \multirow[t]{12}{*}{ Sample mislabeled as 538 in Aq. } & $3 / 6 / 13$ & $528-\mathrm{CO} 2-5.42 \mathrm{mB}-\mathrm{U}$ & 4566 & -37.501 & 0.004 & 3.099 & 0.020 & -54.081 & 0.040 & -0.265 & 0.034 & 0.012 & -44.963 & -2.660 & 0.150 \\
\hline & $3 / 6 / 13$ & 536-Evap+Oz-5.39mB-U & 4575 & -3.042 & 0.005 & 43.663 & 0.009 & 19.312 & 0.026 & 0.192 & 0.025 & 0.009 & 39.907 & 3.123 & 0.126 \\
\hline & $3 / 6 / 13$ & $3808-1$ & 4584 & -3.120 & 0.004 & 39.930 & 0.013 & 15.210 & 0.023 & -0.113 & 0.016 & 0.006 & 31.588 & 2.248 & 0.151 \\
\hline & $3 / 7 / 13$ & carmel chalk & 4600 & -2.151 & 0.003 & 34.691 & 0.020 & 10.863 & 0.039 & -0.192 & 0.044 & 0.015 & 20.482 & 1.509 & 0.128 \\
\hline & $3 / 7 / 13$ & 539-Evap DI Oz-6.15-H & 4614 & -28.125 & 0.003 & 38.084 & 0.019 & -11.887 & 0.028 & -0.903 & 0.026 & 0.009 & 27.407 & 1.846 & 0.183 \\
\hline & $3 / 7 / 13$ & 703-5 inner & 4624 & -7.780 & 0.004 & 37.495 & 0.009 & 8.184 & 0.034 & -0.157 & 0.037 & 0.013 & 26.377 & 1.886 & 0.168 \\
\hline & $3 / 7 / 13$ & 703-5 inner & 4632 & -7.807 & 0.005 & 37.291 & 0.023 & 7.971 & 0.022 & -0.141 & 0.031 & 0.011 & 26.164 & 2.074 & 0.143 \\
\hline & $3 / 7 / 13$ & $3803-4$ & 4641 & -4.186 & 0.005 & 39.568 & 0.016 & 13.763 & 0.043 & -0.154 & 0.032 & 0.011 & 30.628 & 2.016 & 0.130 \\
\hline & $3 / 8 / 13$ & $\mathrm{CM}$ & 4649 & 2.224 & 0.005 & 37.039 & 0.012 & 17.223 & 0.018 & -0.444 & 0.021 & 0.008 & 25.248 & 1.620 & 0.151 \\
\hline & $3 / 8 / 13$ & $3808-1$ & 4657 & -3.136 & 0.007 & 39.956 & 0.020 & 15.214 & 0.015 & -0.119 & 0.015 & 0.005 & 31.712 & 2.318 & 0.135 \\
\hline & $3 / 8 / 13$ & $3806-1$ & 4666 & -4.026 & 0.006 & 39.615 & 0.020 & 14.012 & 0.036 & -0.109 & 0.022 & 0.008 & 31.072 & 2.358 & 0.184 \\
\hline & $3 / 8 / 13$ & $3806-1$ & 4666 & -4.026 & 0.006 & 39.615 & 0.020 & 14.012 & 0.036 & -0.109 & 0.022 & 0.008 & 31.072 & 2.358 & 0.184 \\
\hline
\end{tabular}




\begin{tabular}{|c|c|c|c|c|c|c|c|c|c|c|c|c|c|c|c|}
\hline & $3 / 8 / 13$ & $703-2$ & 4674 & -3.055 & 0.013 & 40.006 & 0.013 & 15.441 & 0.025 & -0.023 & 0.025 & 0.009 & 32.138 & 2.635 & 0.171 \\
\hline & $3 / 8 / 13$ & 542 Evap DI + Oz 5.8- UH & 4683 & -28.243 & 0.005 & 40.216 & 0.012 & -9.134 & 0.032 & -0.097 & 0.025 & 0.009 & 31.947 & 2.158 & 0.078 \\
\hline & $3 / 9 / 13$ & $\mathrm{CM}$ & 4699 & 2.306 & 0.008 & 37.128 & 0.026 & 17.392 & 0.037 & -0.445 & 0.025 & 0.009 & 25.561 & 1.753 & 0.111 \\
\hline \multirow[t]{32}{*}{7 acquisitions } & $3 / 9 / 13$ & 545 Evap DI UH 4719 & 4719 & -3.661 & 0.003 & 45.889 & 0.005 & 18.313 & 0.025 & 0.180 & 0.075 & 0.027 & 44.048 & 2.488 & 1.010 \\
\hline & $3 / 10 / 13$ & Carmel Chalk & 4740 & -2.160 & 0.003 & 34.705 & 0.008 & 10.891 & 0.027 & -0.170 & 0.022 & 0.008 & 20.533 & 1.531 & 0.167 \\
\hline & $3 / 10 / 13$ & 533-tank CO2-5.65mB-H & 4749 & -37.398 & 0.005 & 3.780 & 0.014 & -54.177 & 0.021 & -1.161 & 0.023 & 0.008 & -43.883 & -2.886 & 0.170 \\
\hline & $3 / 10 / 13$ & 544-Evap DI+Oz-6.32mB-UH & 4758 & -3.649 & 0.004 & 45.917 & 0.005 & 20.952 & 0.024 & 0.188 & 0.021 & 0.007 & 44.053 & 2.793 & 0.098 \\
\hline & $3 / 10 / 13$ & 540-Evap DI+Oz-6.97mB-H & 4767 & -27.936 & 0.004 & 40.123 & 0.008 & -9.712 & 0.021 & -0.893 & 0.015 & 0.005 & 31.304 & 1.709 & 0.136 \\
\hline & $03 / 11 / 13$ & 542-Evap Dloz-6.52mB-UH & 4789 & -4.021 & 0.006 & 46.413 & 0.013 & 21.074 & 0.032 & 0.183 & 0.018 & 0.006 & 45.122 & 2.871 & 0.058 \\
\hline & $3 / 11 / 13$ & 543-Evap Dloz-6.33mB-UH & 4798 & -3.636 & 0.003 & 46.152 & 0.009 & 21.222 & 0.015 & 0.211 & 0.016 & 0.006 & 44.547 & 2.817 & 0.098 \\
\hline & $5 / 19 / 13$ & SPEL-2-8-E & 7205 & -9.672 & 0.007 & 32.512 & 0.012 & 1.230 & 0.018 & -0.333 & 0.017 & 0.006 & 16.254 & 1.602 & 0.235 \\
\hline & $\begin{array}{l}5 / 19 / 13 \\
5 / 20 / 13 \\
5 / 20 / 13\end{array}$ & $\begin{array}{c}596 \text { Evap DI oz 6.37mB UH } \\
579 \text { Evap DI oz UH } \\
\text { Carmel Chalk }\end{array}$ & $\begin{array}{l}7215 \\
7264 \\
7272\end{array}$ & $\begin{array}{l}-4.173 \\
-4.081 \\
-2.150\end{array}$ & $\begin{array}{l}0.003 \\
0.006 \\
0.007\end{array}$ & $\begin{array}{l}46.394 \\
45.408 \\
34.801\end{array}$ & $\begin{array}{l}0.007 \\
0.021 \\
0.016\end{array}$ & $\begin{array}{l}20.849 \\
20.041 \\
10.893\end{array}$ & $\begin{array}{l}0.032 \\
0.033 \\
0.039\end{array}$ & $\begin{array}{r}0.130 \\
0.208 \\
-0.271\end{array}$ & $\begin{array}{l}0.031 \\
0.018 \\
0.027\end{array}$ & $\begin{array}{l}0.011 \\
0.006 \\
0.010\end{array}$ & $\begin{array}{l}45.409 \\
43.947 \\
20.793\end{array}$ & $\begin{array}{l}3.184 \\
3.669 \\
1.601\end{array}$ & $\begin{array}{l}0.088 \\
0.278 \\
0.115\end{array}$ \\
\hline & $5 / 21 / 13$ & $\mathrm{CM}$ & 7306 & 2.340 & 0.027 & 37.114 & 0.037 & 17.335 & 0.062 & -0.519 & 0.025 & 0.009 & 25.803 & 2.017 & 0.155 \\
\hline & $5 / 21 / 13$ & 598 bonedry $6.7 \mathrm{mB}$ uh & 7343 & -37.430 & 0.002 & 3.539 & 0.009 & -53.672 & 0.029 & -0.352 & 0.025 & 0.009 & -44.390 & -2.936 & 0.071 \\
\hline & $5 / 22 / 13$ & Carmel Chalk & 7351 & -2.174 & 0.009 & 34.743 & 0.016 & 10.816 & 0.031 & -0.267 & 0.031 & 0.011 & 20.543 & 1.468 & 0.143 \\
\hline & $5 / 23 / 13$ & Carmel Chalk & 7416 & -2.491 & 0.002 & 34.881 & 0.012 & 10.634 & 0.026 & -0.278 & 0.025 & 0.009 & 20.921 & 1.573 & 0.136 \\
\hline & $5 / 23 / 13$ & 603 Evap DI ox $6.1 \mathrm{mB}$ UH & 7456 & -3.839 & 0.004 & 46.620 & 0.007 & 21.449 & 0.022 & 0.175 & 0.021 & 0.007 & 46.044 & 3.360 & 0.103 \\
\hline & $5 / 24 / 13$ & $\mathrm{CM}$ & 7465 & 2.288 & 0.005 & 37.170 & 0.011 & 17.359 & 0.029 & -0.500 & 0.024 & 0.009 & 25.679 & 1.788 & 0.182 \\
\hline & $5 / 24 / 13$ & Carmel Chalk & 7473 & -2.200 & 0.004 & 34.811 & 0.011 & 10.866 & 0.021 & -0.261 & 0.016 & 0.006 & 20.779 & 1.567 & 0.159 \\
\hline & $5 / 28 / 13$ & $\mathrm{CM}$ & 7683 & 2.329 & 0.005 & 37.120 & 0.012 & 17.332 & 0.028 & -0.518 & 0.019 & 0.007 & 25.533 & 1.741 & 0.126 \\
\hline & $5 / 30 / 13$ & 601 Bonedry 5.82mB UH & 7754 & -37.640 & 0.008 & 2.906 & 0.011 & -54.452 & 0.031 & -0.322 & 0.033 & 0.012 & -45.502 & -2.840 & 0.289 \\
\hline & $5 / 30 / 13$ & $\mathrm{CM}$ & 7762 & 2.266 & 0.002 & 37.128 & 0.009 & 17.275 & 0.025 & -0.521 & 0.024 & 0.008 & 25.753 & 1.942 & 0.155 \\
\hline & $5 / 31 / 13$ & 614 Evap + Oz 6.46 mB - H & 7798 & -3.794 & 0.003 & 45.417 & 0.009 & 19.333 & 0.019 & -0.772 & 0.019 & 0.007 & 43.063 & 2.801 & 0.119 \\
\hline & $6 / 1 / 13$ & 616 Bonedry $6.35 \mathrm{mB}$ Heated & 7830 & -37.531 & 0.003 & 4.095 & 0.012 & -54.115 & 0.031 & -1.283 & 0.029 & 0.010 & -43.941 & -3.571 & 0.123 \\
\hline & $6 / 2 / 13$ & 611 bonedry ?mB UH & 7860 & -37.490 & 0.002 & 3.241 & 0.007 & -54.041 & 0.028 & -0.378 & 0.030 & 0.011 & -45.264 & -3.258 & 0.132 \\
\hline & $6 / 3 / 13$ & 609Bone6.11UH & 7918 & -37.605 & 0.003 & 3.332 & 0.009 & -54.053 & 0.017 & -0.369 & 0.020 & 0.007 & -44.925 & -3.082 & 0.190 \\
\hline & $6 / 4 / 13$ & $\mathrm{CM}$ & 7976 & 2.314 & 0.002 & 37.129 & 0.009 & 17.328 & 0.022 & -0.516 & 0.020 & 0.007 & 25.710 & 1.898 & 0.127 \\
\hline & $6 / 5 / 13$ & Bonedry $6.51 \mathrm{mB}-\mathrm{H}$ & 8049 & -37.511 & 0.003 & 4.096 & 0.010 & -54.110 & 0.030 & -1.297 & 0.022 & 0.008 & -44.104 & -3.742 & 0.139 \\
\hline & $6 / 6 / 13$ & 168 Evap DI Oz 6.58 mB H & 8060 & -3.698 & 0.002 & 45.367 & 0.003 & 19.369 & 0.025 & -0.780 & 0.021 & 0.007 & 43.021 & 2.856 & 0.119 \\
\hline & $6 / 6 / 13$ & Carmel Chalk & 8078 & -2.185 & 0.003 & 34.699 & 0.010 & 10.747 & 0.027 & -0.283 & 0.028 & 0.010 & 20.548 & 1.557 & 0.100 \\
\hline & $6 / 6 / 13$ & Carmel Chalk & 8087 & -2.136 & 0.002 & 34.725 & 0.007 & 10.807 & 0.027 & -0.296 & 0.024 & 0.009 & 20.533 & 1.492 & 0.120 \\
\hline & $6 / 7 / 13$ & $\mathrm{CM}$ & 8095 & 2.295 & 0.004 & 37.097 & 0.008 & 17.263 & 0.033 & -0.530 & 0.030 & 0.011 & 25.430 & 1.686 & 0.113 \\
\hline & $6 / 8 / 13$ & 617 Bonedry $6.11 \mathrm{mB}$ UH & 8146 & -37.783 & 0.004 & 3.505 & 0.010 & -54.064 & 0.027 & -0.378 & 0.029 & 0.010 & -44.810 & -3.306 & 0.070 \\
\hline & $6 / 9 / 13$ & $\mathrm{CM}$ & 8231 & 2.329 & 0.014 & 37.020 & 0.030 & 17.244 & 0.057 & -0.506 & 0.021 & 0.007 & 25.261 & 1.669 & 0.080 \\
\hline & $6 / 10 / 13$ & 172 Bonedry - $6.41 \mathrm{mB}-\mathrm{H}$ & 8260 & -37.595 & 0.002 & 3.761 & 0.008 & -54.483 & 0.026 & -1.270 & 0.025 & 0.009 & -44.877 & -3.885 & 0.193 \\
\hline \multirow[t]{3}{*}{ Acid change (2 dummy run prior) } & $6 / 10 / 13$ & TV03 & 8271 & 3.390 & 0.002 & 30.286 & 0.005 & 11.802 & 0.023 & -0.251 & 0.019 & 0.007 & 11.049 & 0.721 & 0.121 \\
\hline & $6 / 11 / 13$ & TV01 & 8312 & 2.471 & 0.002 & 30.246 & 0.007 & 10.867 & 0.027 & -0.253 & 0.028 & 0.010 & 11.022 & 0.775 & 0.120 \\
\hline & $6 / 11 / 13$ & $703-2$ & 8347 & -1.401 & 0.007 & 42.295 & 0.006 & 19.244 & 0.025 & -0.121 & 0.024 & 0.008 & 36.858 & 2.800 & 0.056 \\
\hline
\end{tabular}




\begin{tabular}{|c|c|c|c|c|c|c|c|c|c|c|c|c|c|c|}
\hline $6 / 12 / 13$ & $\mathrm{CM}$ & 8355 & 2.329 & 0.002 & 37.146 & 0.008 & 17.356 & 0.030 & -0.519 & 0.029 & 0.010 & 25.574 & 1.732 & 0.114 \\
\hline $6 / 12 / 13$ & $694-3$ & 8363 & -0.352 & 0.002 & 39.471 & 0.006 & 17.398 & 0.030 & -0.181 & 0.025 & 0.009 & 30.702 & 2.259 & 0.123 \\
\hline $6 / 12 / 13$ & $148-2$ & 8371 & -4.183 & 0.004 & 39.873 & 0.012 & 14.099 & 0.017 & -0.125 & 0.019 & 0.007 & 31.573 & 2.347 & 0.112 \\
\hline $6 / 12 / 13$ & Bonedry $6.12 \mathrm{UH}$ & 8380 & -37.590 & 0.002 & 3.089 & 0.008 & -54.292 & 0.024 & -0.390 & 0.025 & 0.009 & -45.718 & -3.430 & 0.125 \\
\hline $6 / 12 / 13$ & $\mathrm{CM}$ & 8391 & 2.314 & 0.003 & 37.156 & 0.005 & 17.371 & 0.034 & -0.500 & 0.038 & 0.013 & 25.541 & 1.680 & 0.123 \\
\hline $6 / 12 / 13$ & $694-3$ & 8407 & -0.394 & 0.004 & 39.452 & 0.007 & 17.337 & 0.026 & -0.181 & 0.021 & 0.008 & 30.591 & 2.188 & 0.094 \\
\hline $6 / 13 / 13$ & TV01 & 8431 & 2.441 & 0.024 & 30.187 & 0.044 & 10.798 & 0.078 & -0.234 & 0.018 & 0.006 & 10.998 & 0.868 & 0.140 \\
\hline $6 / 13 / 13$ & $148-2$ & 8440 & -4.440 & 0.002 & 40.170 & 0.005 & 14.158 & 0.027 & -0.109 & 0.023 & 0.008 & 32.139 & 2.327 & 0.146 \\
\hline $6 / 13 / 13$ & $3806-1$ & 8451 & -3.470 & 0.003 & 40.384 & 0.006 & 15.260 & 0.033 & -0.170 & 0.029 & 0.010 & 32.615 & 2.373 & 0.147 \\
\hline $6 / 13 / 13$ & TV01 & 8467 & 2.585 & 0.001 & 30.238 & 0.007 & 10.965 & 0.033 & -0.258 & 0.029 & 0.010 & 11.015 & 0.782 & 0.086 \\
\hline $6 / 13 / 13$ & 703-2 & 8475 & -1.423 & 0.002 & 42.345 & 0.005 & 19.290 & 0.025 & -0.105 & 0.022 & 0.008 & 36.941 & 2.782 & 0.105 \\
\hline $6 / 14 / 13$ & $148-2$ & 8491 & -4.685 & 0.002 & 40.034 & 0.008 & 13.754 & 0.038 & -0.137 & 0.036 & 0.013 & 31.909 & 2.367 & 0.146 \\
\hline $6 / 14 / 13$ & 169 Bonedry $-6.15 \mathrm{mB}-\mathrm{H}$ & 8502 & -37.446 & 0.004 & 4.229 & 0.008 & -53.899 & 0.033 & -1.275 & 0.034 & 0.012 & -43.963 & -3.859 & 0.171 \\
\hline $6 / 14 / 13$ & Carmel Chalk & 8524 & -2.120 & 0.002 & 34.858 & 0.005 & 10.974 & 0.024 & -0.278 & 0.020 & 0.007 & 20.760 & 1.458 & 0.122 \\
\hline $6 / 15 / 13$ & TV03 & 8566 & 3.138 & 0.002 & 30.272 & 0.005 & 11.542 & 0.026 & -0.252 & 0.026 & 0.009 & 11.152 & 0.851 & 0.131 \\
\hline $\begin{array}{l}6 / 16 / 13 \\
6 / 16 / 13\end{array}$ & $\begin{array}{c}\mathrm{CM} \\
\text { Bonedry } 6.26 \mathrm{mBUH}\end{array}$ & $\begin{array}{l}8607 \\
8624\end{array}$ & $\begin{array}{c}1.939 \\
-37.596\end{array}$ & $\begin{array}{l}0.004 \\
0.002\end{array}$ & $\begin{array}{c}36.897 \\
3.063\end{array}$ & $\begin{array}{l}0.010 \\
0.007\end{array}$ & & $\begin{array}{l}0.025 \\
0.034\end{array}$ & $\begin{array}{l}-0.510 \\
-0.376\end{array}$ & $\begin{array}{l}0.024 \\
0.036\end{array}$ & $\begin{array}{l}0.008 \\
0.013\end{array}$ & $\begin{array}{r}25.153 \\
-45.895\end{array}$ & $\begin{array}{c}1.802 \\
-3.562\end{array}$ & $\begin{array}{l}0.060 \\
0.082\end{array}$ \\
\hline $6 / 18 / 13$ & $\mathrm{CM}$ & 8702 & 2.268 & 0.005 & 37.114 & 0.010 & 17.266 & 0.017 & -0.518 & 0.020 & 0.007 & 25.672 & 1.890 & 0.135 \\
\hline $6 / 18 / 13$ & 618 Bonedry $6.03 \mathrm{mB} \cup \mathrm{H}$ & 8738 & -37.570 & 0.010 & 3.097 & 0.009 & -54.238 & 0.045 & -0.360 & 0.048 & 0.017 & -45.573 & -3.293 & 0.323 \\
\hline $6 / 18 / 13$ & TV03 & 8746 & 3.274 & 0.003 & 30.357 & 0.004 & 11.801 & 0.020 & -0.211 & 0.017 & 0.006 & 11.368 & 0.898 & 0.113 \\
\hline $6 / 19 / 13$ & Carmel Chalk & 8780 & -2.017 & 0.003 & 35.099 & 0.005 & 11.323 & 0.030 & -0.269 & 0.028 & 0.010 & 21.396 & 1.615 & 0.138 \\
\hline $6 / 20 / 13$ & TV03 & 8807 & 3.305 & 0.019 & 30.289 & 0.036 & 11.737 & 0.057 & -0.236 & 0.022 & 0.008 & 11.140 & 0.805 & 0.111 \\
\hline $6 / 20 / 13$ & 170 Bonedry $6.47 \mathrm{H}$ & 8817 & -37.732 & 0.003 & 3.690 & 0.002 & -54.683 & 0.029 & -1.272 & 0.031 & 0.011 & -45.001 & -3.872 & 0.119 \\
\hline $\begin{array}{l}6 / 20 / 13 \\
6 / 20 / 13\end{array}$ & $\begin{array}{l}3806-1 \text { (run) (8833) } \\
694-13\end{array}$ & $\begin{array}{l}8833 \\
8841\end{array}$ & $\begin{array}{l}-3.557 \\
-0.697\end{array}$ & $\begin{array}{l}0.002 \\
0.003\end{array}$ & $\begin{array}{l}40.346 \\
39.427\end{array}$ & $\begin{array}{l}0.005 \\
0.006\end{array}$ & $\begin{array}{l}15.150 \\
17.027\end{array}$ & $\begin{array}{l}0.026 \\
0.015\end{array}$ & $\begin{array}{l}-0.157 \\
-0.170\end{array}$ & $\begin{array}{l}0.023 \\
0.015\end{array}$ & $\begin{array}{l}0.008 \\
0.005\end{array}$ & $\begin{array}{l}32.518 \\
30.730\end{array}$ & $\begin{array}{l}2.353 \\
2.371\end{array}$ & $\begin{array}{l}0.114 \\
0.098\end{array}$ \\
\hline $6 / 21 / 13$ & $146-6$ & 8849 & -3.271 & 0.003 & 41.053 & 0.005 & 16.198 & 0.030 & -0.094 & 0.027 & 0.010 & 34.114 & 2.538 & 0.108 \\
\hline $6 / 21 / 13$ & 3806-01 (run 2) & 8857 & -3.528 & 0.005 & 40.341 & 0.006 & 15.172 & 0.025 & -0.159 & 0.029 & 0.010 & 32.604 & 2.446 & 0.136 \\
\hline $6 / 21 / 13$ & $\mathrm{CM}$ & 8865 & 2.325 & 0.005 & 37.109 & 0.007 & 17.318 & 0.029 & -0.516 & 0.029 & 0.010 & 25.637 & 1.865 & 0.103 \\
\hline $6 / 21 / 13$ & 694-3 milled & 8873 & -0.292 & 0.001 & 39.014 & 0.007 & 16.955 & 0.033 & -0.225 & 0.027 & 0.009 & 29.746 & 2.210 & 0.141 \\
\hline $6 / 21 / 13$ & $3806-1$ (run 1) & 8892 & -3.541 & 0.002 & 40.277 & 0.008 & 15.084 & 0.029 & -0.171 & 0.030 & 0.010 & 32.453 & 2.422 & 0.154 \\
\hline $6 / 21 / 13$ & $3806-1$ (run 2) & 8900 & -3.726 & 0.013 & 40.291 & 0.024 & 14.903 & 0.038 & -0.183 & 0.021 & 0.007 & 32.377 & 2.321 & 0.092 \\
\hline $6 / 22 / 13$ & $694-13$ & 8909 & -0.709 & 0.003 & 39.439 & 0.008 & 14.899 & 0.024 & -0.148 & 0.063 & 0.022 & 30.587 & 1.933 & 0.785 \\
\hline $6 / 22 / 13$ & $\mathrm{CM}$ & 8917 & 2.326 & 0.002 & 37.161 & 0.006 & 17.373 & 0.014 & -0.515 & 0.013 & 0.005 & 25.634 & 1.761 & 0.132 \\
\hline $6 / 22 / 13$ & 703-7 (run 1) & 8925 & -2.994 & 0.004 & 39.783 & 0.006 & 15.135 & 0.028 & -0.164 & 0.024 & 0.009 & 31.417 & 2.365 & 0.128 \\
\hline $6 / 22 / 13$ & 703-7 (run 2) & 8933 & -3.186 & 0.002 & 39.726 & 0.007 & 14.880 & 0.036 & -0.174 & 0.032 & 0.011 & 31.234 & 2.298 & 0.158 \\
\hline $\begin{array}{l}6 / 22 / 13 \\
6 / 22 / 13\end{array}$ & $\begin{array}{c}703-7 \text { (run 3) } \\
694-13\end{array}$ & $\begin{array}{l}8941 \\
8949\end{array}$ & $\begin{array}{l}-3.194 \\
-0.748\end{array}$ & $\begin{array}{l}0.004 \\
0.002\end{array}$ & $\begin{array}{l}39.647 \\
39.412\end{array}$ & $\begin{array}{l}0.011 \\
0.007\end{array}$ & $\begin{array}{l}14.797 \\
16.952\end{array}$ & $\begin{array}{l}0.028 \\
0.034\end{array}$ & $\begin{array}{l}-0.171 \\
-0.179\end{array}$ & $\begin{array}{l}0.024 \\
0.031\end{array}$ & $\begin{array}{l}0.009 \\
0.011\end{array}$ & $\begin{array}{l}31.177 \\
30.598\end{array}$ & $\begin{array}{l}2.393 \\
2.272\end{array}$ & $\begin{array}{l}0.233 \\
0.127\end{array}$ \\
\hline $6 / 22 / 13$ & 9 Bonedry $\mathrm{CO} 26.79$ & 8958 & -37.585 & 0.001 & 3.512 & 0.009 & -53.903 & 0.026 & -0.413 & 0.026 & 0.009 & -45.045 & -3.566 & 0.127 \\
\hline $6 / 22 / 13$ & $\mathrm{CM}$ & 8966 & 2.222 & 0.002 & 37.126 & 0.006 & 17.253 & 0.024 & -0.497 & 0.025 & 0.009 & 25.604 & 1.800 & 0.127 \\
\hline $6 / 23 / 13$ & 694-3 (run2) & 8982 & -0.419 & 0.003 & 39.432 & 0.009 & 17.292 & 0.041 & -0.181 & 0.040 & 0.014 & 30.749 & 2.381 & 0.088 \\
\hline
\end{tabular}




\begin{tabular}{|c|c|c|c|c|c|c|c|c|c|c|c|c|c|c|}
\hline $6 / 23 / 13$ & $148-2$ (run 1) & 8991 & -4.469 & 0.003 & 40.106 & 0.008 & 14.051 & 0.031 & -0.123 & 0.026 & 0.009 & 32.204 & 2.512 & 0.133 \\
\hline $\begin{array}{l}6 / 23 / 13 \\
6 / 23 / 13\end{array}$ & $\begin{array}{c}148-2 \text { (run 2) } \\
\text { CM }\end{array}$ & $\begin{array}{l}8999 \\
9008\end{array}$ & $\begin{array}{l}-4.398 \\
2.317\end{array}$ & $\begin{array}{l}0.004 \\
0.003\end{array}$ & $\begin{array}{l}40.104 \\
37.219\end{array}$ & $\begin{array}{l}0.013 \\
0.009\end{array}$ & $\begin{array}{l}14.123 \\
17.454\end{array}$ & $\begin{array}{l}0.029 \\
0.042\end{array}$ & $\begin{array}{l}-0.118 \\
-0.484\end{array}$ & $\begin{array}{l}0.020 \\
0.037\end{array}$ & $\begin{array}{l}0.007 \\
0.013\end{array}$ & $\begin{array}{l}32.039 \\
25.847\end{array}$ & $\begin{array}{l}2.357 \\
1.858\end{array}$ & $\begin{array}{l}0.097 \\
0.075\end{array}$ \\
\hline $6 / 23 / 13$ & $703-2$ (old) & 9024 & -1.884 & 0.283 & 40.084 & 3.185 & 17.603 & 0.027 & -0.130 & 0.057 & 0.020 & 34.518 & 2.636 & 0.086 \\
\hline $6 / 24 / 13$ & 703-2 (new) & 9032 & -1.506 & 0.004 & 42.214 & 0.010 & 19.068 & 0.017 & -0.113 & 0.016 & 0.006 & 36.734 & 2.835 & 0.071 \\
\hline $6 / 24 / 13$ & $146-6$ (old) & 9040 & -2.383 & 0.008 & 40.632 & 0.008 & 16.616 & 0.038 & -0.128 & 0.038 & 0.014 & 33.492 & 2.742 & 0.112 \\
\hline $6 / 24 / 13$ & TV03 & 9048 & 3.339 & 0.015 & 30.388 & 0.008 & 11.873 & 0.028 & -0.233 & 0.022 & 0.008 & 11.732 & 1.198 & 0.130 \\
\hline $\begin{array}{l}6 / 24 / 13 \\
6 / 24 / 13\end{array}$ & $\begin{array}{c}\text { 180-Bonedry-5.94-UH } \\
694-13\end{array}$ & $\begin{array}{l}9059 \\
9067\end{array}$ & $\begin{array}{c}-37.525 \\
-0.734\end{array}$ & $\begin{array}{l}0.003 \\
0.006\end{array}$ & $\begin{array}{c}2.836 \\
39.377\end{array}$ & $\begin{array}{l}0.008 \\
0.009\end{array}$ & $\begin{array}{r}-54.437 \\
16.927\end{array}$ & $\begin{array}{l}0.012 \\
0.038\end{array}$ & $\begin{array}{l}-0.351 \\
-0.183\end{array}$ & $\begin{array}{l}0.010 \\
0.032\end{array}$ & $\begin{array}{l}0.003 \\
0.011\end{array}$ & $\begin{array}{c}-46.278 \\
30.620\end{array}$ & $\begin{array}{c}-3.512 \\
2.362\end{array}$ & $\begin{array}{l}0.128 \\
0.099\end{array}$ \\
\hline $6 / 24 / 13$ & $3808-5$ & 9075 & -5.981 & 0.003 & 39.179 & 0.007 & 11.562 & 0.024 & -0.208 & 0.023 & 0.008 & 30.049 & 2.211 & 0.148 \\
\hline $6 / 24 / 13$ & $3808-4$ & 9084 & -2.948 & 0.007 & 40.023 & 0.009 & 15.378 & 0.022 & -0.205 & 0.019 & 0.007 & 31.904 & 2.376 & 0.159 \\
\hline $6 / 25 / 13$ & $3808-3$ & 9094 & -4.540 & 0.002 & 39.436 & 0.008 & 13.228 & 0.022 & -0.210 & 0.018 & 0.006 & 30.790 & 2.430 & 0.142 \\
\hline $6 / 25 / 13$ & $\mathrm{CM}$ & 9102 & 2.302 & 0.002 & 37.197 & 0.008 & 17.383 & 0.016 & -0.517 & 0.017 & 0.006 & 25.863 & 1.916 & 0.111 \\
\hline $6 / 25 / 13$ & Adamossi (run 1) & 9111 & 1.832 & 0.010 & 43.420 & 0.013 & 23.569 & 0.020 & -0.099 & 0.025 & 0.009 & 39.388 & 3.068 & 0.180 \\
\hline $6 / 25 / 13$ & Adamossi (run 2) & 9121 & 1.818 & 0.001 & 43.432 & 0.005 & 23.569 & 0.051 & -0.097 & 0.050 & 0.018 & 39.329 & 2.988 & 0.124 \\
\hline $6 / 26 / 13$ & Adamossi (run 3) & 9129 & 1.826 & 0.004 & 43.450 & 0.009 & 23.586 & 0.043 & -0.107 & 0.044 & 0.015 & 39.421 & 3.042 & 0.166 \\
\hline $6 / 26 / 13$ & 620 Evap + Oz - $5.97 \mathrm{mB}-\mathrm{H}$ & 9138 & -3.777 & 0.005 & 42.539 & 0.009 & 16.480 & 0.018 & -0.781 & 0.019 & 0.007 & 37.081 & 2.556 & 0.094 \\
\hline $6 / 27 / 13$ & 703-2 (old) run 2 & 9189 & -2.073 & 0.003 & 41.139 & 0.011 & 17.450 & 0.034 & -0.103 & 0.026 & 0.009 & 34.539 & 2.780 & 0.159 \\
\hline $6 / 27 / 13$ & TV03 & 9197 & 3.326 & 0.002 & 30.248 & 0.020 & 11.707 & 0.031 & -0.246 & 0.022 & 0.008 & 11.334 & 1.076 & 0.169 \\
\hline $6 / 27 / 13$ & 146-6 (new) (run1) & 9208 & -3.426 & 0.004 & 40.994 & 0.006 & 15.954 & 0.020 & -0.128 & 0.017 & 0.006 & 34.605 & 3.128 & 0.113 \\
\hline $6 / 27 / 13$ & 146-6 (new) (run2) & 9216 & -3.415 & 0.002 & 41.073 & 0.009 & 16.072 & 0.028 & -0.099 & 0.033 & 0.012 & 34.243 & 2.626 & 0.081 \\
\hline $6 / 28 / 13$ & $694-13$ (run 2) & 9232 & -0.658 & 0.004 & 39.485 & 0.009 & 16.977 & 0.359 & -0.187 & 0.019 & 0.007 & 31.386 & 2.521 & 0.094 \\
\hline $6 / 28 / 13$ & $\mathrm{CM}$ & 9240 & 2.345 & 0.009 & 37.161 & 0.011 & 17.373 & 0.026 & -0.532 & 0.025 & 0.009 & 25.938 & 2.058 & 0.137 \\
\hline $6 / 29 / 13$ & 171 Bonedry $6.13 \mathrm{mB} \mathrm{H}$ & 9311 & -37.636 & 0.008 & 3.763 & 0.017 & -54.542 & 0.024 & -1.291 & 0.029 & 0.010 & -44.817 & -3.824 & 0.156 \\
\hline $7 / 3 / 13$ & 178 Bonedry- $6.53 \mathrm{mB}-\mathrm{UH}$ & 9529 & -37.864 & 0.002 & 3.192 & 0.007 & -54.477 & 0.027 & -0.416 & 0.027 & 0.009 & -46.048 & -3.976 & 0.122 \\
\hline $7 / 5 / 13$ & 176 Bonedry $5.97 \mathrm{mB}-\mathrm{H}$ & 9641 & -37.763 & 0.004 & 2.908 & 0.011 & -55.473 & 0.036 & -1.282 & 0.039 & 0.014 & -47.058 & -4.469 & 0.121 \\
\hline $7 / 7 / 13$ & 183 Bonedry $\mathrm{CO} 26.33 \mathrm{mB} \mathrm{UH}$ & 9747 & -37.759 & 0.002 & 2.383 & 0.008 & -55.136 & 0.038 & -0.396 & 0.034 & 0.012 & -47.661 & -4.057 & 0.099 \\
\hline $7 / 10 / 13$ & 185 Bonedry CO2- $6.51 \mathrm{mB}-\mathrm{UH}$ & 9940 & -37.856 & 0.002 & 2.651 & 0.007 & -54.975 & 0.021 & -0.400 & 0.027 & 0.010 & -47.040 & -3.939 & 0.139 \\
\hline $7 / 11 / 13$ & 623 Evap DI oz $5.77 \mathrm{mB}$ UH & 9981 & -3.834 & 0.004 & 46.136 & 0.015 & 20.890 & 0.020 & 0.092 & 0.018 & 0.007 & 45.307 & 3.579 & 0.097 \\
\hline $7 / 14 / 13$ & Bonedry174 CO2 6.38mB H & 10156 & -37.638 & 0.002 & 4.306 & 0.004 & -54.059 & 0.040 & -1.330 & 0.040 & 0.014 & -44.135 & -4.190 & 0.155 \\
\hline $7 / 16 / 13$ & 624 Evap DI + Oz 5.9mB UH & 10271 & -3.777 & 0.023 & 46.662 & 0.047 & 21.448 & 0.087 & 0.072 & 0.024 & 0.008 & 46.375 & 3.595 & 0.142 \\
\hline $7 / 18 / 13$ & 626 Evap.DI+oz $6.17 \mathrm{mB} \mathrm{H}$ & 10372 & -3.884 & 0.002 & 46.050 & 0.016 & 19.889 & 0.041 & -0.756 & 0.030 & 0.011 & 45.033 & 3.480 & 0.125 \\
\hline $7 / 18 / 13$ & 177 Bonedry $6.22 \mathrm{mB} \cup \mathrm{H}$ & 10409 & -37.540 & 0.003 & 2.941 & 0.010 & -54.417 & 0.015 & -0.421 & 0.019 & 0.007 & -46.543 & -3.996 & 0.174 \\
\hline $7 / 20 / 13$ & 627 Evap DI+Oz $6.11 \mathrm{mB} \mathrm{H}$ & 10440 & -3.860 & 0.002 & 45.605 & 0.008 & 19.481 & 0.021 & -0.745 & 0.024 & 0.008 & 44.038 & 3.379 & 0.150 \\
\hline $7 / 21 / 13$ & 184 Bonedry $6.25 \mathrm{UH}$ & 10462 & -37.362 & 0.004 & 3.866 & 0.014 & -53.358 & 0.021 & -0.420 & 0.023 & 0.008 & -44.654 & -3.860 & 0.140 \\
\hline $7 / 28 / 13$ & $632 \mathrm{Evap} \mathrm{DI}+\mathrm{Oz} 6.35 \mathrm{mB}$ UH & 10484 & -3.814 & 0.003 & 46.665 & 0.007 & 21.466 & 0.051 & 0.122 & 0.043 & 0.015 & 46.285 & 3.503 & 0.193 \\
\hline
\end{tabular}


Fig. S1

\begin{tabular}{lccccc} 
& \multicolumn{2}{c}{ from multiple regression } & \multicolumn{2}{c}{ emp. Transfer function } \\
line & \multicolumn{2}{c}{ intercept - observed } & intercept - theoretical & slope & 1.033396 \\
$250 \mathrm{C}$ & 0.05506 & 0.9252 & intercept & 0.868301 \\
$1000 \mathrm{oC}$ & & -0.8145 & 0.0266 & &
\end{tabular}
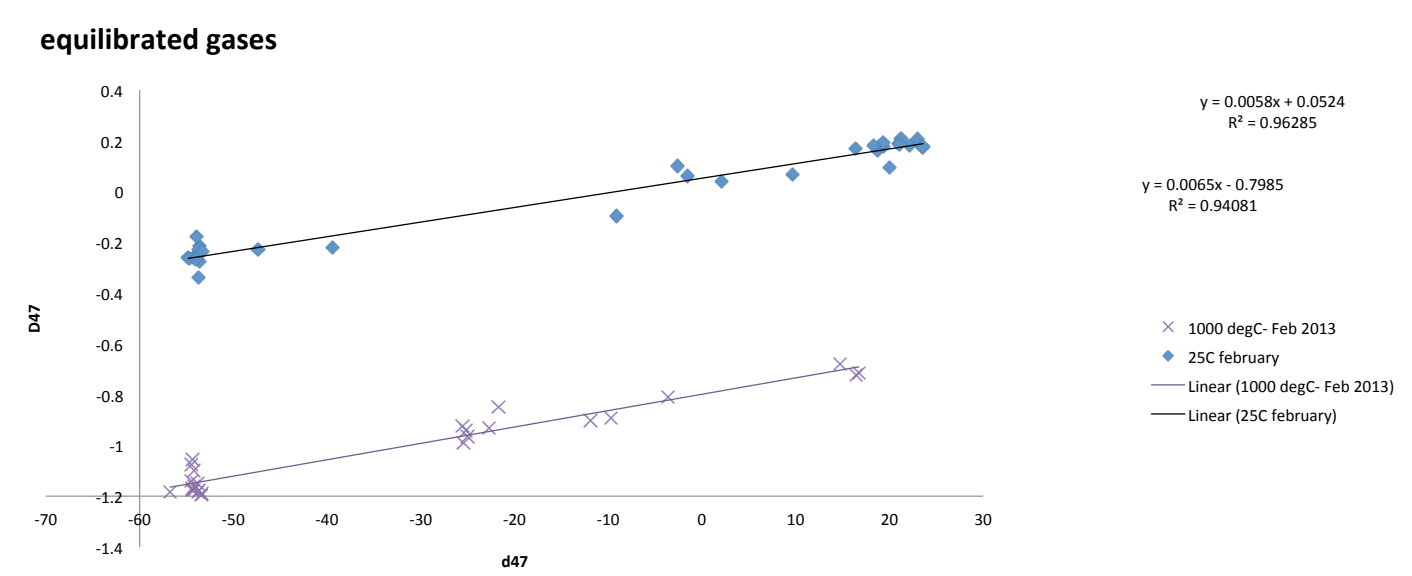
Fig. S2

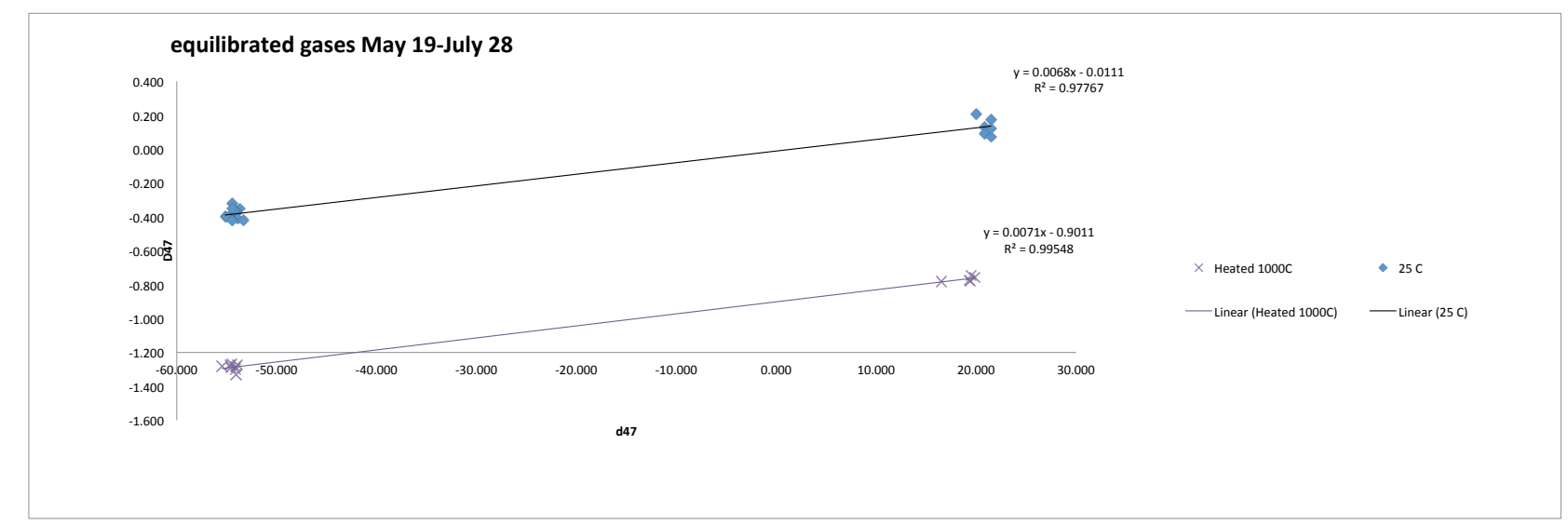

Population 1

\begin{tabular}{|c|c|c|c|c|c|}
\hline & & May 19 - July 28 & & & \\
\hline line & & intercept - observed & intercept - theoretical & emp. Transfer function & \\
\hline $250 \mathrm{C}$ & & -0.007724 & 0.9252 & slope & $\begin{array}{l}1.001475577 \\
0.932935397\end{array}$ \\
\hline $\begin{array}{c}10000 \mathrm{C} \\
\text { common slope }\end{array}$ & 0.006952 & -0.905 & 0.0266 & intercept & 0.932935397 \\
\hline
\end{tabular}

